\title{
Propiedades de aleaciones Ag-Pd para usos odontológicos $^{(\bullet)}$
}

\author{
J. Basualto ${ }^{(*)}$, C. Barceló $\left.{ }^{*}\right)$ y A. Gaete ${ }^{(* *)}$ \\ Resumen En la búsqueda de aleaciones para usos odontológicos, económicamente alternativas, se trabaja con \\ tres aleaciones Ag-Pd complejas, del tipo Ag-Pd-Au-Cu-Otros, dos aleaciones experimentales y un \\ producto comercial. Se determinan las propiedades mecánicas y químicas de estas aleaciones y se \\ hace un análisis comparativo de los resultados. El costo, factor importante, se establece con relación \\ a los materiales del producto comercial. Se determina la dureza Vickers de las aleaciones Ag-Pd con \\ diversos tratamientos térmicos, lo que permite mostrar su versatilidad en cuanto a usos restauradores \\ y clasificarlas según norma. Se determina su comportamiento electroquímico y la resistencia a la \\ pigmentación, lo que permite comparar las tendencias exhibidas. Se concluye que las propiedades \\ químicas son aceptables cuando se compara con aleaciones odontológicas de referencia. Se \\ instalaron en boca aleaciones experimentales A1 y A2, hace más de un año, con resultados \\ satisfactorios hasta ahora, lo que valida las predicciones de laboratorio.
}

Palabras clave: Aleaciones Ag-Pd. Aplicaciones en odontología. Métodos electroquímicos. Pigmentación.

\section{Properties of Ag-Pd alloys for odontological uses}

\begin{abstract}
In this search for economically alternative alloys for odontological uses, three complex Ag-Pd alloys, $\mathrm{Ag}-\mathrm{Pd}-\mathrm{Au}-\mathrm{Cu}$ type, are studied: two experimental alloys and one commercial product. Chemical and mechanical properties were obtained for these alloys and a comparative analysis of these results was made. The cost, important factor, is established in relation to the materials cost of the commercial product. Vickers hardness values obtained from Ag-Pd alloys with different heat treatments allow to show their versatility in relation to restorative uses and to classify them according to standard practice. Electrochemical and tarnish behavior were obtained that allow to compare the trends. It is concluded that chemical properties are acceptable, when they are compared with odontological alloys of reference. Experimental alloys A1 y A2 were installed in mouth, over a year ago, with satisfactory results until now, which validate previous laboratory predictions.
\end{abstract}

Keywords: Ag-Pd alloys. Odontologigal applications. Electrochemical methods. Tarnish.

\section{INTRODUCCIÓN}

Las aleaciones para usos dentales, con alto contenido de metales nobles en su composición, tienen actualmente costos prohibitivos para una fracción importante de los pacientes. Como solución a este problema, se plantea el estudio de aleaciones económicamente alternativas. Sin embargo, no es fácil introducir en el mercado nuevos productos cuando los usuarios desconocen sus propiedades, cualida-

(•) Trabajo recibido el día 3 de octubre de 1995.

(*) Dpto. Ciencia de Materiales. Universidad Santa María. Avda. España 1680. Valparaíso (Chile).

(**) Dpto. Operatoria Dental. Facultad de Odontología. Universidad de Valparaíso. Playa Ancha. Valparaíso (Chile). des y limitaciones. Estudios anteriores muestran la factibilidad de uso en boca de aleaciones $\mathrm{Cu}-\mathrm{Al}$ (1 y 2), de muy bajo costo, siendo determinante para la aceptación sus propiedades químicas y mecánicas. Por otro lado, se están usando con éxito productos comerciales Ag-Pd, con un contenido medio de metales nobles, aunque su costo es todavía algo elevado.

Si se comparan las aleaciones antes mencionadas, $\mathrm{Cu}-\mathrm{Al}$ y Ag-Pd, en cuanto a propiedades mecánicas y químicas, se puede señalar que las primeras, siendo importantes, no establecen diferencias apreciables entre ambas familias; sin embargo, las propiedades químicas son muy diferentes. Se observa un comportamiento de las aleaciones $\mathrm{Ag}-\mathrm{Pd}$, de costo medio, cercano al de las aleaciones de mayor contenido de metales nobles, y un comportamiento 
inferior, aunque todavía aceptable, de las aleaciones $\mathrm{Cu}-\mathrm{Al}$. Si esta diferencia de comportamiento se asocia a diferencias de precios, nace la idea de investigar aleaciones complejas $\mathrm{Cu}-\mathrm{Al}$ que, aunque elevando los costos, mejoren sus propiedades químicas; siendo la otra alternativa disminuir los costos de las aleaciones Ag-Pd, con el riesgo de empeorar estas propiedades, pero manteniéndolas todavía en un rango clínicamente aceptable.

En este trabajo se comprueba esta segunda alternativa, es decir, la posibilidad de obtener aleaciones $\mathrm{Ag}-\mathrm{Pd}$ complejas, que presenten una adecuada relación costo/calidad, aptas para usos dentales, a un costo de materiales inferior al de aleaciones comerciales importadas, de uso frecuente en nuestro medio.

El comportamiento clínico de las aleaciones dentales se correlaciona muy bien con su comportamiento electroquímico (3), por lo que resulta interesante el siguiente detalle. En éste se ha considerado la corriente de inicio de pasivación como elemento comparativo.

\begin{tabular}{|l|l|}
\hline \multicolumn{1}{|c|}{ Grupo de aleaciones } & $\begin{array}{l}\text { Comportamiento } \\
\text { electroquímico }\end{array}$ \\
\hline $\begin{array}{l}\text { A. - Aleaciones base oro y grupo del platino } \\
\text { B. - Aleaciones Ag-Pd con alto contenido en } \\
\text { metales nobles }\end{array}$ & $\begin{array}{l}\text { Excelente } \\
\text { Muy bueno } \\
\text { C. - Aleaciones Cu-Al complejas } \\
\text { D. - Aleaciones Cu-Zn }\end{array}$ \\
& Bueno-Aceptable \\
& Aceptable- \\
& Deficiente \\
\hline
\end{tabular}

En términos muy generales, desde el punto de vista del comportamiento electroquímico, se comprende que aquellas aleaciones que presentan elevadas densidades de corriente tienen una baja resistencia a la corrosión y, por lo tanto, tendrán también un mal comportamiento clínico. Por el contrario, aleaciones que presenten bajas densidades de corriente de corrosión mostrarán un buen comportamiento en boca. Entre los grupos A, B y C puede haber diferencias de un orden de magnitud entre sus correspondientes densidades de corriente, expresadas en $\mathrm{mA} / \mathrm{cm}^{2}$.

Las aleaciones de interés para la investigación se ubican entre los grupos $\mathrm{B}$ y $\mathrm{C}$, es decir, en un rango intermedio entre el comportamiento aceptable de las mejores aleaciones $\mathrm{Cu}-\mathrm{Al}$ y el buen comportamiento de las Ag-Pd, aun con contenidos medios de metales nobles. Se postula como posible encontrar aleaciones Ag-Pd que, con un menor contenido de metales nobles, presenten todavía una buena resistencia a la corrosión, siendo aptas para su uso en boca. Esto estaría avalado por el comportamiento clínico aceptable mostrado por las aleaciones $\mathrm{Cu}$ $\mathrm{Al}$, ubicadas en el rango superior de su grupo.

Trabajando con aleaciones Ag-Pd complejas del tipo Ag-Pd-Au-Cu (4), se observó la posibilidad de desarrollar aleaciones de un costo, en materiales, que podría llegar a ser la mitad del de un producto comercial utilizado como referencia. En este trabajo, se han seleccionado dos aleaciones experimentales y una comercial para hacer un estudio comparativo de sus propiedades químicas y mecánicas, se discute la influencia de los distintos elementos y su posible aplicación odontológica.

\section{MATERIALES Y MÉTODOS}

Se trabaja con tres aleaciones Ag-Pd complejas, no excediendo de un $20 \%$ el contenido de metales nobles en las aleaciones experimentales. De esta manera, su costo con relación al producto comercial de referencia es inferior a 1. Estos detalles, de composición y costos, se presentan en la tabla I.

El costo es uno de los factores considerados para definir la composición de las aleaciones experimentales y, por lo tanto, resulta de interés presentarlo en dicha tabla. Se ha determinado sólo en función del valor comercial de los componentes de cada aleación, referido al costo de materiales del producto comercial (AC), no incluyendo costos de fabricación y otros.

Las aleaciones madre se obtienen mediante técnicas de fundición por inducción y las muestras experimentales por colada centrífuga, de manera similar a la obtención de los colados dentales, trabajando con el método de la cera perdida. Las muestras, en estado bruto de colada, se someten a controles iniciales de dureza y, posteriormente, a tratamientos térmicos de homogeneización y de endurecimiento. Las muestras finales, en forma de pequeños discos de $1 \mathrm{~cm}^{2}$ de sección y $1 \mathrm{~mm}$ de altura, se preparan metalográficamente: se montan en resina transóptica (lucita), se desbastan con lijas de carburo de silicio y luego se pulen en pasta de diamante de hasta $0,25 \mu \mathrm{m}$, terminando con un pulido final en alúmina de $0,05 \mu \mathrm{m}$. De esta manera, estando las muestras en condiciones de observación microscópica, se controla su estado superficial, se determina su microdureza Vickers y se someten a ensayos para determinar sus propiedades químicas, a partir de sus curvas de polarización y del

TABLA I.- Composición nominal y costo relativo (CR) de las aleaciones investigadas

TABLE I.- Nominal composition and relative cost $(C R)$ of the studied alloys

\begin{tabular}{|c|c|c|c|c|c|c|}
\hline Aleación & \multicolumn{5}{|c|}{ Composición, \% en peso } & Costo \\
\hline Núm. & $\mathrm{Ag}$ & $\mathrm{Pd}$ & $\mathrm{Au}$ & $\mathrm{Cu}$ & Otros & $\mathrm{CR}$ \\
\hline $\mathrm{A} 1$ & 79 & 15 & - & 5 & 1 & 0,46 \\
$\mathrm{~A} 2$ & 79 & 15 & 5 & - & 1 & 0,82 \\
$\mathrm{AC}$ & 67 & 23 & 5 & - & 5 & 1,00 \\
\hline
\end{tabular}


grado de pigmentación. Se utiliza saliva artificial Fusayama modificada (5) para determinar las curvas de polarización y una solución acuosa de sulfuro de sodio (6) al 0,5\%, como medio agresivo para los ensayos de pigmentación.

Para restauraciones dentales es necesario usar aleaciones con diferentes propiedades mecánicas, que suelen clasificarse en cuatro grupos, con relación a su dureza. Según lo establecido por la Asociación Dental Americana (ADA), para aleaciones de metales nobles, base oro, estos grupos corresponden a aleaciones blandas, medianas, duras y extraduras. A las aleaciones Ag-Pd investigadas, tratadas y preparadas según se describe anteriormente, se les midió microdureza Vickers, lo que permite su clasificación dentro de los tipos ADA señalados, se utiliza como referencia los valores de dureza Vickers especificados para estos grupos (7).

Si se pretende predecir el comportamiento clínico de una aleación dental, se debe poner especial atención en la determinación de sus propiedades químicas, corrosión y pigmentación. La tendencia a la corrosión, que exhiben estas aleaciones, se determina mediante técnicas electroquímicas de polarización potenciodinámica. Utilizando como electrólito saliva artificial y manteniendo la temperatura del baño alrededor de $37^{\circ} \mathrm{C}$, se obtienen las curvas de polarización anódica de las aleaciones investigadas. Estas se determinan en condiciones desaireadas, efectuando un barrido total de $1 \mathrm{~V}$ a una velocidad de $30 \mathrm{mV} / \mathrm{min}$. Las curvas así obtenidas permiten comparar el comportamiento electroquímico de las aleaciones investigadas, según las densidades de corriente que se generan durante el barrido de potencial a que se someten. Por otro lado, la pigmentación se determina utilizando un método desarrollado por Tuccillo y Nielsen (6), el cual consiste en una rueda acrílica, o porta-muestras, que contiene 8 de ellas, en moldes de lucita de 1 pulg. $(25,4 \mathrm{~mm})$ de diámetro, y que gira a baja velocidad, entre 1 y 3 r.p.m., permitiendo que las muestras queden alternativamente expuestas a la solución de sulfuro de sodio, al $0,5 \%$, y al aire. Trabajando a 1,5 r.p.m., en cada revolución las muestras están sumergidas, aproximadamente, en el medio agresivo $9 \mathrm{~s}, 2 \mathrm{~s}$ semisumergidas y $29 \mathrm{~s}$ al aire. Los ensayos tienen una duración de $72 \mathrm{~h}$, midiéndose, al término de los cuales, la pérdida de brillo de la muestra mediante técnicas de reflexión de la luz, con un microscopio metalográfico. En estas condiciones, el grado de pigmentación, alcanzado por las aleaciones investigadas, se define porcentualmente como una relación entre la luz reflejada por la superficie inicial, en estado de pulido, y la reflexión de la superficie alterada durante el ensayo, siendo el grado de pigmentación $=100 *\left(I_{\mathrm{r}}-I_{\mathrm{m}}\right) / I_{\mathrm{r}}$. donde, $I_{\mathrm{r}}$ representa la reflexión de la superficie pulida e $I_{\mathrm{m}}$ la reflexión de la superficie pigmentada.

\section{RESULTADOS EXPERIMENTALES}

\subsection{Tratamientos térmicos y microdureza}

Los diversos requerimientos mecánicos de las prótesis dentales hacen deseable que su resistencia, medida a través de la dureza, pueda ser ajustada mediante tratamientos térmicos. Las aleaciones AgPd suelen utilizarse en condición blanda o endurecida; la condición blanda, utilizada como referencia, se obtiene mediante calentamiento a $750{ }^{\circ} \mathrm{C}$ por $1 \mathrm{~h}$ $\mathrm{y}$ posterior enfriamiento brusco en agua. El endurecimiento, a partir de la condición blanda, se logra mediante tratamientos de envejecimiento; para estas aleaciones los máximos valores de dureza se suelen alcanzar para temperaturas entre 250 y $400{ }^{\circ} \mathrm{C}$ (8). En este trabajo se fijó un rango algo más amplio, entre 200 y $450{ }^{\circ} \mathrm{C}$ y el tiempo en $1 \mathrm{~h}$, considerando poco práctico la utilización de tiempos mayores.

En las muestras con tratamiento térmico y preparadas metalográficamente, utilizando una carga de $300 \mathrm{~g}$, se determinó la microdureza Vickers correspondiente. Los niveles de dureza alcanzados por las aleaciones envejecidas, a partir de la condición blanda u homogeneizada, se muestran en la figura 1. Cada punto indicado en esta figura representa el promedio de 5 mediciones, la máxima desviación estándar de estos resultados alcanzó a 2,5 unidades Vickers. El producto comercial parece alcanzar su máxima dureza para la temperatura de tratamiento más elevada, coincidiendo el valor obtenido con la máxima dureza, $140 \mathrm{HV}$, especificada por el fabricante. Por otro lado, considerando los valores ADA de dureza Vickers (7), en la figura 2 se comparan las cifras Vickers (HV) experimentales, correspondientes a los valores extremos de las condiciones blandas y duras.

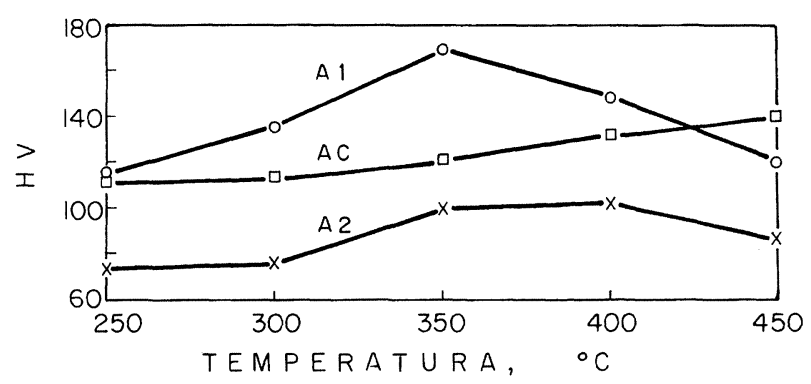

FIG. 1.- Microdureza Vickers de muestras envejecidas durante $1 \mathrm{~h}$, a temperaturas comprendidas entre 250 y $450{ }^{\circ} \mathrm{C}$. Inicialmente, todas las muestras se calentaron a $750{ }^{\circ} \mathrm{C}$, permaneciendo $1 \mathrm{~h}$ a esta temperatura y enfriándose después bruscamente en agua.

FIG. 1.- Vickers microhardness of specimens aged for $1 \mathrm{~h}$ at temperatures in the range 250 to $450{ }^{\circ} \mathrm{C}$. Initially, the specimens were heated for $1 \mathrm{~h}$ at $750{ }^{\circ} \mathrm{C}$ and then water quenched. 


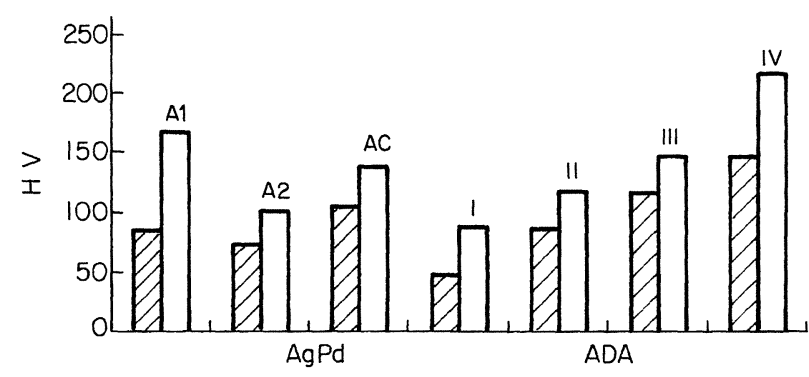

FIG. 2.- Valores extremos de microdureza Vickers de las aleaciones experimentales, obtenidos mediante tratamiento térmico, en comparación con los rangos de dureza de los diferentes tipos de la clasificación ADA.

FIG. 2.- Maximum and minimum Vickers microhardness values of experimental alloys, obtained by heat treatment, compared with hardness ranges of different types of ADA classification.

\subsection{Curvas de polarización}

El posible comportamiento frente a la corrosión, de las aleaciones que se consideran de interés, se puede analizar a partir de las curvas de polarización anódica que éstas exhiben. Agrupando las curvas obtenidas para cada condición de tratamiento, se pueden comparar las tendencias presentadas por las distintas aleaciones, utilizando como electrólito la saliva artificial.

La figura 3 muestra las curvas representativas de las tres aleaciones investigadas en su condición blanda. El comportamiento de estas aleaciones queda enmarcado por el excelente comportamiento de una aleación $\mathrm{Au}-\mathrm{Cu}(88 \mathrm{Au}-12 \mathrm{Cu})$ y el, compara-

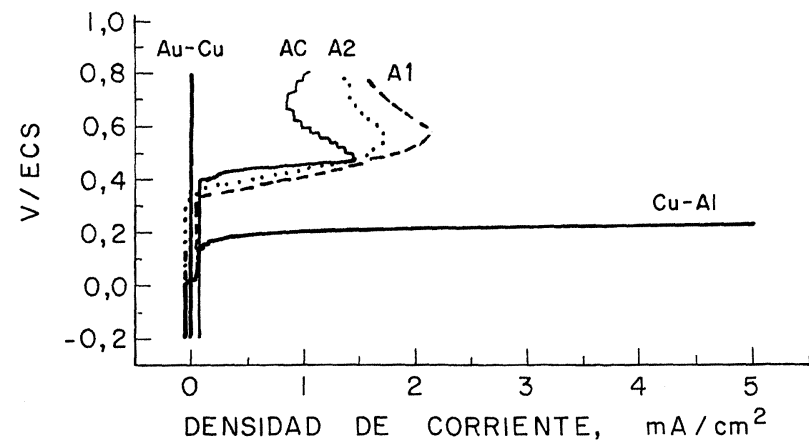

FIG. 3.- Curvas de polarización anódica en saliva artificial, a $37^{\circ} \mathrm{C}$, de las aleaciones Ag-Pd investigadas, en su condición blanda. Se comparan con las curvas de las aleaciones de referencia, $\mathrm{Au}-\mathrm{Cu}$ y $\mathrm{Cu}-\mathrm{Al}$.

FIG. 3.- Anodic polarization curves, in artificial saliva at $37{ }^{\circ} \mathrm{C}$, for the Ag-Pd investigated alloys, in the soft condition. The comparison is made with curves of reference alloys $\mathrm{Au}-\mathrm{Cu}$ and $\mathrm{Cu}-\mathrm{Al}$. tivamente, mal comportamiento exhibido por una aleación base $\mathrm{Cu}-9 \mathrm{Al}$, ambas utilizadas como material dental restaurador.

La figura 4 muestra las curvas representativas de las tres aleaciones investigadas, en su condición dura. Al igual que en la figura anterior, el comportamiento de estas aleaciones queda enmarcado por la aleación base oro y la de base cobre, usadas como referencia.

\subsection{Ensayos de pigmentación}

La pérdida de brillo o cambio de color que presenta una aleación, producto de reacciones químicas entre el metal y un medio agresivo, se conoce como pigmentación. Para evaluar este fenómeno, en este caso se utilizó como medio agresivo una solución de sulfuro de sodio al $0,5 \%$. Se hace la determinación según el método antes señalado, obteniéndose los resultados que se presentan en la figura 5 .

A continuación se representa gráficamente el comportamiento frente a la pigmentación de las aleaciones Ag-Pd. El grado de pigmentación se expresa como el porcentaje de pérdida de brillo entre la muestra inicial, pulida metalográficamente, y la muestra pigmentada. El gráfico permite comparar directamente el comportamiento de las aleaciones investigadas, en estado bruto de colada, condición considerada la más desfavorable para este ensayo.

\section{DISCUSIÓN}

En cuanto a las propiedades mecánicas, en este caso representadas por la dureza, los resultados obtenidos permiten apreciar que el contenido de cobre es determinante para la obtención de los más

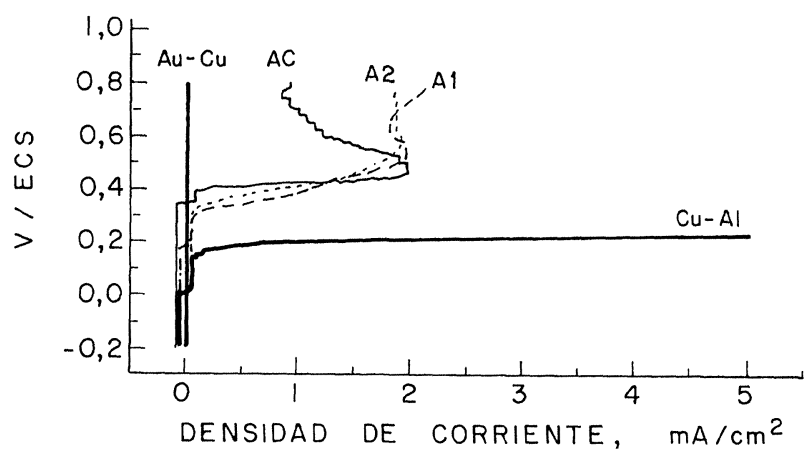

FIG. 4.- Igual que figura 3; en este caso, con las aleaciones experimentales en su condición dura.

FIG. 4.- Same as figure 3, with the experimental alloys in the hard condition. 


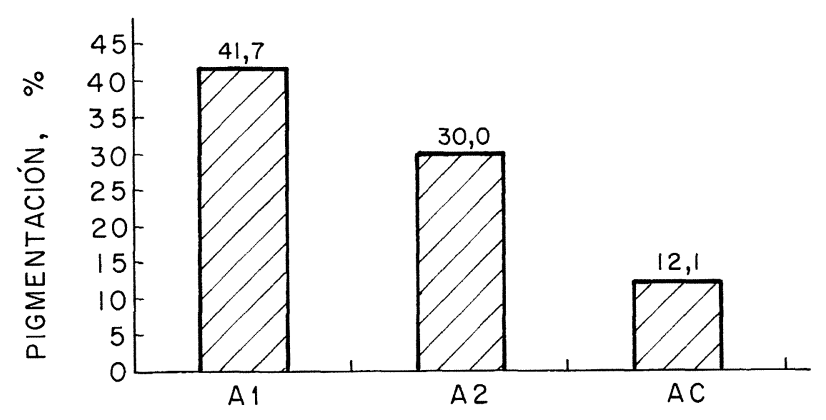

FIG. 5.- Grado de pigmentación (\%) de las aleaciones investigadas, en su condición de colada, ensayadas en sulfuro de sodio a $37^{\circ} \mathrm{C}$, durante $72 \mathrm{~h}$.

FIG. 5.- Degree of tarnish (\%) of the investigated alloys in the as cast condition, tested in sodium sulfide at $37^{\circ} \mathrm{C}$, for $72 \mathrm{~h}$.

altos valores de dureza. La aleación experimental 1 (A1), con un $5 \% \mathrm{Cu}$, presenta los valores de dureza más elevados; el producto comercial $\mathrm{AC}$, que también tiene elementos endurecedores, exhibe una dureza intermedia, y la aleación A2, que no contiene cobre, muestra una dureza menor. A pesar de lo anterior, las durezas medias obtenidas para estas aleaciones muestran una cierta tendencia a su clasificación dentro de los tipos II y III de la ADA. Se corroboran así los resultados anteriores en cuanto a que las variaciones de composición influyen en la dureza de estas aleaciones, pero no llegan a ser limitantes para su uso odontológico. Según clasificación ADA, las aleaciones "blandas" son recomendadas para restauraciones menores, donde cabe esperar bajas solicitaciones mecánicas. Las aleaciones "medias" son una buena alternativa para situaciones en el límite del uso de aleaciones blandas y duras. Las aleaciones "duras" son las indicadas para restauraciones mayores, puentes, coronas y otros. Las aleaciones "extraduras" se utilizan para grandes puentes y otras piezas que requieran una elevada resistencia mecánica.

La buena correlación que existe entre el comportamiento químico y clínico de las aleaciones para usos dentales, hace que los factores asociados con la resistencia a la corrosión sean los más importantes a considerar, para aceptar o contraindicar el uso de estas aleaciones. En este caso, el comportamiento frente a la corrosión se asocia a parámetros electroquímicos, obtenidos del análisis de las curvas de polarización. Un buen comportamiento frente a la corrosión no sólo está asociado a bajas densidades de corriente, sino también a elevados potenciales de inicio de la corrosión. En las figuras 3 y 4, los conjuntos de curvas de polarización permiten apreciar comparativamente los factores considerados $\left(E_{\text {cor }} \mathrm{e}\right.$ $\left.I_{\mathrm{ap}}\right)$. En general, las aleaciones que exhiben una ele- vada densidad de corriente de inicio de la pasivación, $I_{\text {ap }}$, muestran también un potencial menor de inicio de corrosión. Los comportamientos extremos están fijados por la aleación base cobre, el peor, y por la aleación de oro, el mejor. Para las aleaciones $\mathrm{Ag}-\mathrm{Pd}$, aunque las diferencias en los distintos estados no son significativas, las tendencias exhibidas en su condición blanda muestran que el mejor comportamiento lo ofrece la aleación AC comercial; la aleación experimental A2 presenta un comportamiento intermedio, con relación a la A1, es determinante el mayor contenido de metales nobles, ya que ésta contiene un $5 \%$ de oro; el contenido de plata es el mismo para las aleaciones A1 y A2 (9). La aleación $\mathrm{A} 1$, en la cual el oro ha sido reemplazado por el cobre, presenta un comportamiento algo inferior al de la aleación A2. Ambas presentan comportamientos inferiores al del producto comercial, que tiene un mayor contenido de metales nobles y, por lo tanto, un menor contenido de plata. En la condición dura, desde el punto de vista de la $I_{\text {ap }}$, no se aprecian las tendencias antes señaladas. Sin embargo, AC parece presentar un comportamiento mejor, si se considera el $E_{\text {cor }}$ algo más elevado y una tendencia a la pasivación más pronunciada.

Si se consideran las tendencias antes señaladas, como representativas del comportamiento electroquímico y se comparan con el costo relativo de cada aleación, es posible apreciar que el mejor comportamiento es el de la aleación comercial, que tiene un costo relativo unitario; el peor lo presenta la aleación A1, pero sin estar demasiado alejado de la otra aleación experimental A2, ni de la aleación comercial. Por lo tanto, A1 con un comportamiento aceptable y con el menor costo relativo parece presentar el mejor compromiso costo-calidad; la aleación A2 muestra un comportamiento intermedio, algo mejor que A1, pero su costo relativo es mayor.

En el estado inicial, las aleaciones madres con mayor contenido de plata presentan también un mayor brillo pero, expuestas a un medio agresivo, la situación se invierte, mostrando la influencia negativa de un elevado contenido de plata, en la resistencia a la pigmentación de estas aleaciones (10).

Para iguales contenidos de plata y paladio, el oro es determinante en cuanto al aspecto inicial de la aleación, observándose cambios importantes cuando se reemplaza oro por cobre; esto resulta evidente si se comparan las aleaciones A1 y A2, ambas con $79 \% \mathrm{Ag}$ y $15 \%$ Pd; la aleación A2 con $5 \% \mathrm{Au}$ presenta un brillo muy superior al observado en la aleación A1, en la cual se ha reemplazado el oro por cobre. Lo que sucede con el aspecto original, aleación madre, se proyecta al estado post-ensayo de pigmentación, es decir, la aleación con cobre presenta una menor resistencia a la pigmentación 
que la aleación con oro. Sin embargo, en las piezas dentales se pueden lograr brillos más o menos equivalentes en ambas aleaciones, aunque esto significa un mayor trabajo para la aleación con cobre.

\section{CONCLUSIONES}

Si se trabaja con aleaciones base Ag-Pd, lo que se podría llamar límites teóricos de costo estarían fijados, el inferior por la plata, con un costo relativo 0,1 y el superior por el paladio, con un costo relativo 3 , ambos valores referidos a la aleación comercial (AC). Mecánicamente, tampoco es satisfactorio el comportamiento de los metales puros; por lo tanto, resulta obvio que un mejor compromiso se debe lograr con una adecuada combinación de estos elementos, a lo cual se debe agregar el efecto de otros elementos aleantes y de los tratamientos térmicos.

Prácticamente, en esta investigación, el límite superior de costo ha sido fijado a partir de un producto Ag-Pd comercial (AC), de probado buen comportamiento clínico, al que se le asignó un costo relativo unitario. Desde el punto de vista económico, la aleación más atractiva es la experimental A1, ya que no contiene oro; sin embargo, la aleación A2, con oro, cumple también con los objetivos de la investigación, aunque tiene un costo relativo más cercano a la unidad.

El contenido de oro y de cobre influye de diversas maneras en las propiedades generales de estas aleaciones pero, en los rangos estudiados no parecen determinantes para la aceptación o rechazo de éstas. Sin embargo, las tendencias son claras en cuanto a señalar mejores propiedades mecánicas para la aleación A1 (efecto del cobre) y mejores propiedades químicas para la aleación A2 (efecto del oro). Probablemente, trabajando con aleaciones complejas Ag-Pd-Au-Cu, sea posible encontrar todavía mejores compromisos costo-calidad variando los contenidos de oro y cobre, en el sentido de maximizar los contenidos de cobre y minimizar los de oro.

En cuanto a tratamientos térmicos y propiedades mecánicas asociadas, es determinante el contenido de cobre en la capacidad de endurecimiento de estas aleaciones, lo que se puede apreciar al comparar los máximos valores de dureza obtenidos para la aleación $\mathrm{A} 1$, con $5 \% \mathrm{Cu}$, y la aleación $\mathrm{A} 2$, donde el cobre ha sido reemplazado por el oro. La aleación comercial no contiene cobre pero sí otros elementos que pueden contribuir al endurecimiento ( $\mathrm{Sn}$ y $\mathrm{Zn}$ ), los que le permiten alcanzar una dureza máxima intermedia entre las exhibidas por las aleaciones experimentales. Los rangos de dureza pre- sentados por estas aleaciones en sus diferentes estados de tratamiento muestran que la aleación A1 podría cubrir todos los tipos y usos contemplados por la ADA, y la aleación A2, algo más blanda, sólo los tipos ADA I y II.

Las propiedades químicas, representadas por el comportamiento electroquímico y la pigmentación, muestran una relación directa entre el mejor comportamiento y la cantidad de metales nobles de las aleaciones. Los diferentes comportamientos observados no son directamente indicativos de rechazo de las aleaciones experimentales, ya que son propios de los medios agresivos en los cuales se realizaron los ensayos. Desde un punto de vista comparativo, las aleaciones experimentales presentan un comportamiento intermedio entre aleaciones dentales ricas en metales nobles y otras que no los contienen. Tomando, por ejemplo, la corriente de pasivación como parámetro representativo de la tendencia a los fenómenos de corrosión en boca que exhibirían eștas aleaciones, se puede hacer un análisis simple de su relación comportamiento/costo, resumida de la siguiente manera:

A1: Comportamiento intermedio, costo relativo medio-bajo $(0,46)$

A2: Comportamiento intermedio, costo relativo medio-alto $(0,82)$

AC: Mejor comportamiento, costo relativo elevado $(1,00)$

El comportamiento intermedio de las aleaciones experimentales se considera todavía aceptable para una aleación dental, lo que justifica el interés por proyectar este estudio al campo de la evaluación clínica. Hasta la fecha se han instalado en boca 9 restauraciones, fabricadas con aleaciones A1 y A2, las que han sido y están siendo evaluadas según normativa de calidad de la Asociación Dental de California (ADC). Estas restauraciones llevan más de un año puestas en boca con resultados satisfactorios, según clasificación $\mathrm{ADC}$, lo que estaría avalando la predicción de aceptabilidad basada en los resultados experimentales.

Por otro lado, desde el punto de vista metalúrgico, también se puede proyectar esta investigación, en el sentido de optimizar las propiedades de estas aleaciones mediante tratamientos térmicos y quizás con leves modificaciones de su composición. En todos los casos, se debe complementar esta investigación con una etapa de evaluación clínica, que es el paso final en un estudio de este tipo y que, en definitiva, puede proyectar o contraindicar el uso de una aleación para ser puesta en boca. 


\section{Agradecimiento}

Los autores agradecen a FONDECYT Chile la financiación, mediante el Proyecto 1931142, del presente estudio.

\section{REFERENCIAS}

(1) Basualto, J., Barcelo, C. y Orellana, E. Rev. Fac. Odont. UV, 1 (1), 1991: 15-20.

(2) Mueller, H. Metals Handbook, 9a. De., Vol. 13, 1.3531.354 .

(3) LAUB, L. y StANFord, J. Gold Bull. (1) 1981: 13-18.
(4) Basualto, J., Barcelo, C. y Gaete, A. Rev. Fac. Odont. UV, 1 (5) 1995: 241-247.

(5) Meyer, J. y Nally, J. 11th. Annual Congress of the IADR. Continental European Div. Bruselas, 1974: 1-8.

(6) Tuccillo, J. y Nielsen, J. Prosth. Dent., 25 (6), 1971: 629-637.

(7) Lopez Álvarez, J.L., Técnicas de Laboratorio en Prótesis Fija. Gráficas Matacolor. Madrid, 1987: 154.

(8) Cerovic, D., Stankovic, S., Duuric, B. y Jovanovic M. Metallog. (18) 1985: 395-404.

(9) Jovanovic, M., Lukic, G., Gligic, M. y Lukic, B. Metallog. (19), 1986: 447-459.

(10) Niemi, L. y Holland, R.I. J. Dent. Res. 63 (7), 1984: 1.014-1.018. 